\title{
ALGEBRAS OF AUTOMORPHIC FORMS WITH FEW GENERATORS
}

\author{
BY \\ PHILIP WAGREICH ${ }^{1}$
}

\begin{abstract}
Those finitely-generated Fuchsian groups $G$ for which the graded algebra of automorphic forms $A$ is generated by 2 or 3 elements are classified. In these cases the structure of $A$ is described.
\end{abstract}

Suppose $G$ is a finitely-generated Fuchsian group of the first kind and $A_{G}$ is the (graded) ring of automorphic forms relative to $G$. The purpose of this paper is to give a self-contained proof of the classification of those groups $G$ for which the ring $A_{G}$ is generated (as an algebra over $C$ ) by $\leqslant 3$ elements. The first results in this direction were announced by Dolgachev [D1] who studied the case where $G$ is a triangle group $G_{n_{1}, n_{2}, n_{3}}$. That is, $G$ is the subgroup of orientation-preserving maps of the group generated by reflections in the sides of a hyperbolic triangle with angles $\pi / n_{1}, \pi / n_{2}$ and $\pi / n_{3}$. He showed that exactly 14 triangles have the property that $A_{G}$ is generalized by 3 elements. The remaining groups for which $H_{+} / G$ is compact were studied independently by Dolgachev [D2] and myself. Milnor [M] has studied rings of automorphic forms with fractional weight. This paper will describe the classification for arbitrary $G$. The idea of the proof is elementary and makes heavy use of the fact $A_{G}$ is a graded ring (Definition (1.1)) i.e., $A_{G}=$ $\bigoplus_{k>0} A_{k}$, where $A_{k}$ is the vector space of $k$-forms. Suppose $A_{G}$ is generated as a C-algebra by 3 elements $x_{0}, x_{1}, x_{2}$. Elementary arguments (1.5) show that the generators can be chosen to be homogeneous. Let $x_{i} \in A_{q_{i}}$. We let $S=$ C $\left[X_{0}, X_{1}, X_{2}\right]$ and define a grading on $S$ by letting degree $X_{i}=q_{i}$. Define $\varphi$ : $S \rightarrow A_{G}$ by $\varphi\left(X_{i}\right)=x_{i}$ and let $I=\operatorname{kernel} \varphi$. Then we have

Proposition (2.7). I is a principal ideal.

Now the kernel of a homomorphism of graded algebras is a homogeneous ideal, i.e., $I=\bigoplus_{n=0}^{\infty} I_{n}$ where $I_{n}=I \cap A_{n}$. One can easily show that $I$ is generated by a homogeneous element. Suppose that the element is $f \in S$. Then

$$
f\left(t^{q_{0}} Z_{0}, t^{q_{1}} Z_{1}, t^{q_{2}} Z_{2}\right)=t^{d} f\left(Z_{0}, Z_{1}, Z_{2}\right)
$$

where $d$ is an integer called the (weighted) degree of $f$. Such a polynomial is called a weighted homogeneous polynomial, i.e., homogeneous with respect to the grading of $S$ defined above. We let $R=S / I$. The dimension of $R_{i}$ can be computed easily

Received by the editors November 4, 1977.

1980 Mathematics Subject Classification. Primary 10D15, 30F35; Secondary 14J17.

Key words and phrases. Automorphic form, Fuchsian group, graded ring, Poincaré power series.

${ }^{1}$ Research partially supported by grants from the National Science Foundation. 
in terms of $q_{0}, q_{1}, q_{2}$ and $d$. On the other hand, $\operatorname{dim} A_{i}$ can be computed using the Riemann-Roch theorem.

By equating $\operatorname{dim} R_{i}$ and $\operatorname{dim} A_{i}$ and observing some elementary properties of rings of the form $S / I$ we can eliminate all but a list of 42 types of groups. Direct calculation then gives the structure of $A_{G}$ for that list of groups.

This paper is organized as follows. The first section is devoted to a review of properties of graded rings and the Poincaré power series of a graded ring. In $\$ 2$ we calculate the Poincaré power series of $A_{G}$ and as a consequence develop relations between invariants of $G$ (the signature of $G$ ) and $q_{0}, q_{1}, q_{2}$ and $d$. Then in $\S 3$ we find a list of groups $G$ so that all groups with $A_{G}$ generated by $\leqslant 3$ elements are on the list. The fourth section is devoted to proving that all of the groups on the list are, in fact, generated by 2 or 3 elements.

This paper is essentially self-contained. In a subsequent paper [W2], we will use the theory of singularities of complex surfaces to give more general results on the structure of algebras of automorphic forms.

I would like to thank Irwin Kra and Igor Dolgachev for stimulating correspondence.

1. Graded algebras. Suppose $K$ is a field and $R$ is a $K$-algebra.

Definition (1.1). A grading on $R$ is a collection of $K$-vector subspaces $R_{i}$ of $R$ so that

(i) $R=\bigoplus_{i=-\infty}^{\infty} R_{i}$.

(ii) $R_{i} R_{j} \subset R_{i+j}$.

The $K$-algebra $R$ together with a grading is called a graded $K$-algebra. We say $x \in R$ is homogeneous of degree $i$ if $x \in R_{i}$.

Example (1.2). $R=K\left[X_{0}, \ldots, X_{n}\right]$. Fix integers $q_{0}, \ldots, q_{n}$. Define $R_{i}=K$ subspace generated by $X_{0}^{i_{0}} \cdots X_{n}^{i_{n}}$ so that $i_{0} q_{0}+\cdots+i_{n} q_{n}=i$. Note that

$$
f \in R_{i} \Leftrightarrow f\left(t^{q_{0}} X_{0}, \ldots, t^{q_{n}} X_{n}\right)=t^{i} f\left(X_{0}, \ldots, X_{n}\right),
$$

$t$ an indeterminate. If $q_{0}=q_{1}=\cdots=q_{n}=1$ we get the usual grading on $R$.

Definition (1.3). A homomorphism $\varphi: S \rightarrow R$ of graded algebras is said to be homogeneous of degree $d$ if $\varphi\left(S_{i}\right) \subset R_{i+d}$, for all $i$.

Definition (1.4). We say $R$ is a graded $K$-algebra of finite type if there are homogeneous elements $x_{0}, \ldots, x_{n} \in R$ so that the homomorphism

$$
\varphi: K\left[X_{0}, \ldots, X_{n}\right] \rightarrow R
$$

defined by $\varphi\left(X_{i}\right)=x_{i}$ is onto.

Henceforth we shall assume $R$ is positively graded (i.e., $R_{i}=\{0\}$ for $i<0$ ) and $R_{0}=K$.

(1.5) If we are as before and degree $x_{i}=q_{i}$ and we grade $S=K\left[X_{0}, \ldots, X_{n}\right]$ as in Example (1.2) then $\varphi$ is a graded homomorphism of degree 0. It follows that $I=\operatorname{kernel} \varphi$ is a homogeneous ideal, i.e., $I=\bigoplus_{i} I_{i}$. Thus $I$ is generated by homogeneous elements. Let $\mathrm{m}=\bigoplus_{i>0} R_{i}$. Then $\mathrm{m}$ is a maximal ideal of $R$. The embedding dimension of $R$ is defined to be $K$-dimension of the vector space $\mathrm{m} / \mathrm{m}^{2}$. The algebra $R$ is of finite type if and only if $\operatorname{dim}_{K} \mathrm{~m} / \mathrm{m}^{2}<\infty$ and in that case 
$x_{0}, \ldots, x_{n}$ generate $R$ as a $K$-algebra if and only if their residues $\tilde{x}_{0}, \ldots, \bar{x}_{n} \in$ $\mathrm{m} / \mathrm{m}^{2}$ generate $\mathrm{m} / \mathrm{m}^{2}$ as a $K$ vector space. Note that $\mathrm{m} / \mathrm{m}^{2}$ is a graded vector space and we can choose $n$ homogeneous generators for $\mathrm{m} / \mathrm{m}^{2}$. These lift to $n$ homogeneous algebra generators of $R$. The above discussion shows that the embedding dimension of $R$ is the minimal $n$ so that the algebraic variety $\operatorname{Spec}(R)$ can be embedded in affine $n$-space over $K$.

Definition (1.6). Suppose $R$ is a graded $K$-algebra, $M$ is a graded $R$-module and $a_{i}=\operatorname{dim}_{K} M_{i}<\infty$ for all $i$. Let $\mathscr{P}_{M}(t)=\sum_{i=0}^{\infty} a_{i} t^{i}$, the Poincaré power series of $M$.

We now sketch the calculation of $\mathscr{P}_{R}(t)$ in the case we will be studying.

Proposition (1.7). Suppose $S=K\left[X_{0}, \ldots, X_{n}\right]$ is graded as in Example (1.2), $f \in S_{d}, I=(f)$ and $R=S / I$. Then

(i) $\mathscr{P}_{S}(t)=1 / \prod_{i=0}^{n}\left(1-t^{q_{i}}\right)$,

(ii) $\mathscr{P}_{R}(t)=\left(1-t^{d}\right) / \Pi_{i=0}^{n}\left(1-t^{q_{i}}\right)$.

Proof.

Lemma. Suppose $0 \rightarrow M^{\prime} \stackrel{\varphi^{\prime}}{\rightarrow} M \stackrel{\varphi}{\rightarrow} M^{\prime \prime} \rightarrow 0$ is an exact sequence of graded $S$ modules, $\varphi$ is homogeneous of degree $0, \varphi^{\prime}$ is homogeneous of degree $\alpha$. Then

$$
t^{\alpha} \mathscr{P}_{M^{\prime}}(t)-\mathscr{P}_{M}(t)+\mathscr{P}_{M^{\prime \prime}}(t)=0 .
$$

The proof of the lemma is immediate. To prove (i) one can proceed by induction by considering the exact sequence

$$
0 \rightarrow K\left[X_{0}, \ldots, X_{i}\right] \stackrel{\varphi^{\prime}}{\rightarrow} K\left[X_{0}, \ldots, X_{i}\right] \stackrel{\varphi}{\rightarrow} K\left[X_{0}, \ldots, X_{i-1}\right] \rightarrow 0
$$

where $\varphi^{\prime}(g)=X_{i} g$ and $\varphi$ is defined by $\varphi\left(X_{j}\right)=X_{j}, j \neq i, \varphi\left(X_{i}\right)=0$.

To prove (ii) we use the exact sequence

$$
0 \rightarrow S \stackrel{\varphi^{\prime}}{\rightarrow} S \rightarrow R \rightarrow 0
$$

where $\varphi^{\prime}(g)=f g$.

REMARK. One can show as above that for any graded $K$-algebra of finite type $\mathscr{P}_{R}(t)$ is a rational function [A-M].

In $\S 2$ we will be interested in the principal part of $\mathscr{P}_{R}(t)$.

Proposition (1.8). If $p(t)=\Pi_{i=0}^{n}\left(1-t^{d_{i}}\right) / \Pi_{j=0}^{n+2}\left(1-t^{q_{j}}\right)$, then the principal part of $p$ at $t=1$ is $a /(1-t)^{2}+b /(1-t)$ where

$$
a=\frac{\prod_{i=0}^{n} d_{i}}{\prod_{j=0}^{n+2} q_{j}} \quad \text { and } \quad b=a\left(\sum_{j=0}^{n+2} \frac{q_{j}-1}{2}-\sum_{i=0}^{n} \frac{d_{i}-1}{2}\right) .
$$

Proof.

$$
p(t)=\frac{1}{(1-t)^{2}} \frac{\prod_{i=0}^{n}\left(1+\cdots+t^{d_{i}-1}\right)}{\prod_{j=0}^{n+2}\left(1+\cdots+t^{q_{j}-1}\right)} .
$$


Now let $z=1-\mathrm{t}$. Then $1+\cdots+t^{m-1}=m-m(m-1) z / 2+f(z)$ where $f$ consists of terms of degree $\geqslant 2$ in $z$. Thus

$$
p(t)=\frac{1}{z^{2}} \cdot \prod_{i=0}^{n}\left(d_{i}-\frac{d_{i}\left(d_{i}-1\right)}{2} z\right) \cdot \prod_{j=0}^{n+2} \frac{1}{q_{j}}\left(1+\frac{q_{j}-1}{2} z\right)+g(z)
$$

where $g(z)$ is holomorphic at $z=0$. This gives us the desired result.

Proposition (1.9). Let $f(t)=\left(1-t^{d}\right) /\left(1-t^{a}\right)\left(1-t^{b}\right)\left(1-t^{c}\right)$ where $a, b, c, d$ are positive integers. Suppose $\xi^{e}=1$ and $\xi \neq 1$.

(a) If $e \mid a, e \nmid b, c, d$ then the principal part of $f$ at $t=\xi$ is

$$
\left(-\xi\left(1-\xi^{d}\right) / a\left(1-\xi^{b}\right)\left(1-\xi^{c}\right)\right) \cdot(1 /(1-t)) .
$$

(b) If $e|a, e| b, e \mid d, e \nmid c$ then the principal part of $f$ at $t=\xi$ is

$$
\left(-d \xi / a b\left(1-\xi^{c}\right)\right) \cdot(1 /(1-t))
$$

Proof. Let $z=t-\xi$ and substitute for $t$ in the given function. The principal part is easily calculated.

Definition (1.10). The dimension of a $K$-algebra $R$ is the transcendence degree of the quotient field of $R$ over $K$.

REMARK (1.11). (a) If $R$ is finitely generated over $K$ then $\operatorname{dim} R=$ Krull $\operatorname{dim} R$.

(b) If $R$ is a finitely-generated graded algebra then dimension $R=$ order of pole of $\mathscr{P}_{R}(t)$ at $t=1[\mathbf{A}-\mathbf{M}]$.

Definition (1.12). If $S=K\left[X_{0}, \ldots, X_{n}\right]$ is graded by letting degree $X_{i}=q_{i}>$ 0 , we define $c\left(q_{0}, \ldots, q_{n}\right)_{i}=\operatorname{dim}_{K} S_{i}$ which by Proposition (1.7) equals the coefficient of $t^{i}$ in the power series $1 / \prod_{i=0}^{n}\left(1-t^{q_{i}}\right)$.

When calculating this integer by hand it is frequently helpful to use the recursion formula

$$
c\left(q_{0}, \ldots, q_{n}\right)_{i}=c\left(q_{0}, \ldots, q_{n-1}\right)_{i}+c\left(q_{0}, \ldots, q_{n}\right)_{i-q_{n}}
$$

for all $i$. Also

$$
c(1, \ldots, 1)_{i}=\left(\begin{array}{c}
i+n \\
n
\end{array}\right) .
$$

The calculations in the later parts of this paper were done by using a computer, evaluating $c\left(q_{0}, \ldots, q_{n}\right)_{i}$ by multiplication of the power series $1 /\left(1-t^{q_{i}}\right)=$ $\sum_{k=0}^{\infty} t^{k q_{i}}$.

\section{Automorphic forms.}

(2.1) The Lie group $\operatorname{PSL}(2, \mathbf{R})$ acts on the upper half plane $H_{+}$by $\gamma \cdot z=$ $(a z+b) /(c z+d)$, where $\left(\begin{array}{ll}a & b \\ c & d\end{array}\right)$ is a representative for $\gamma$ and $z \in H_{+}$. Henceforth we shall assume that $G$ is a subgroup of $\operatorname{PSL}(2, \mathbf{R})$, that $G$ acts properly discontinuously on $H_{+}$and that $X=H_{+} / G$ is a compact Riemann surface with a finite number of punctures, i.e., there is a compact Riemann surface $\bar{X}$ and an open immersion of $X=H_{+} / G$ into $\bar{X}$ so that $\bar{X}-X$ consists of a finite number of points. Let $\sigma$ be the number of punctures.

Let $p_{1}, \ldots, p_{r} \in X$ be the points where $H_{+} \rightarrow X$ is branched and let $e_{i}$ be the ramification index over $p_{i}$. We number the $p_{i}$ so that $e_{1} \leqslant e_{2} \leqslant \cdots \leqslant e_{r}$. We let $e_{r+1}=\cdots=e_{r+\sigma}=\infty$. If $g=$ genus $\bar{X}$ then $\left\{g ; \sigma ; e_{1}, \ldots, e_{r}\right\}$ is called the 
signature of $G$. We will also use the notation $\left\{g ; e_{1}, \ldots, e_{r+\sigma}\right\}$ for the signature of $G$. By [K, p. 78] a set of integers as just mentioned arises from a group $G$ as before if and only if

$$
\sigma+\sum_{i=1}^{r}\left(1-\frac{1}{e_{i}}\right)+2 g-2>0 .
$$

Alternately $\sum_{i=1}^{r+o}\left(1-1 / e_{i}\right)+2 g-2>0$.

(2.2) A meromorphic function $f(z)$ on $H_{+}$is said to be an unrestricted automorphic form of weight $k$ for $G$ if

$$
f((a z+b) /(c z+d))=(c z+d)^{2 k} f(z)
$$

for all $\left(\begin{array}{ll}a & b \\ c & d\end{array}\right) \in G$. Equivalently

$$
f(\gamma \cdot z)=(d \gamma / d z)^{-k} f(z)
$$

For any parabolic element of $G$ we can change coordinates so that its fixed point is at $\infty$. Let $A(z)=z+1$ be the generator of the stabilizer of $\infty$. Then $f$ has a Fourier expansion $f(z)=\sum_{n=-\infty}^{\infty} a_{n} e^{2 \pi i n z}$. We say $f$ is entire if $f$ is holomorphic in $H_{+}$and $a_{n}=0$ for $n<0$ (for each parabolic subgroup of $G$ ). We say $f$ is a cusp form if $f$ is entire and $a_{n}=0$ for $n \leqslant 0$ (see [G, II.7] for details).

Define $A_{k}=$ the $\mathrm{C}$-vector space of entire automorphic forms of weight $k, k \geqslant 0$.

$$
C_{k}=\text { the subspace of cusp forms, } \quad k \geqslant 1 .
$$

Define $A_{G}=\bigoplus_{k=0}^{\infty} A_{k}$, the algebra of automorphic forms,

$$
e_{G}=\bigoplus_{k=1}^{\infty} C_{k}, \quad \text { the ideal of cusp forms. }
$$

Then $A_{G}$ is a graded algebra and $C_{G}$ is a homogeneous ideal. If we define $C_{\mathbf{0}}=\mathbf{C}$ and $C_{G}=\bigoplus_{k=0}^{\infty} C_{k}$ we call $C_{G}$ the algebra of cusp forms.

Henceforth, when there can be no confusion we will denote $A_{G}$ by $A$.

ReMARK. In [W2] we prove that $A_{G}$ is a finitely-generated C-algebra. It follows that $C_{G}$ is a finitely-generated ideal. On the other hand $C_{G}$ is not a finitely-generated C-algebra (see (4.1)).

(2.3) The dimension of the vector space $A_{k}$ can be calculated using the Riemann-Roch theorem. For example, one can use the proof in [G, Chapter II, Theorem 1] slightly modified. If $G$ has signature $\left\{g ; \sigma ; e_{1}, \ldots, e_{r}\right\}$ then

$$
\operatorname{dim} A_{k}= \begin{cases}(2 k-1)(g-1)+\sigma k+\sum_{i=1}^{r}\left[k\left(1-\frac{1}{e_{i}}\right)\right] & \text { if } k>1 \text { or } \sigma>0 \\ g & \text { if } k=1 \text { and } \sigma=0 \\ 1 & \text { if } k=0 .\end{cases}
$$

The symbol $[x]$ denotes the largest integer $\leqslant x$.

(2.4) An elementary calculation using (2.3) shows that the Poincaré power series (1.6) of $A$ is

$$
\mathcal{P}_{A}=\frac{2 g-2+\sigma}{(1-t)^{2}}+\frac{3-3 g-\sigma}{(1-t)}+\sum_{i=1}^{r} P_{e_{i}}(t)+\delta_{\sigma+1,1} t+g
$$


where $P_{e_{i}}(t)=\sum_{k=0}^{\infty}\left[k\left(1-1 / e_{i}\right)\right] t^{k}$ and $\delta$ denotes the Kronecker delta.

We shall be interested in the partial fraction decomposition of $\boldsymbol{P}_{\boldsymbol{e}}$. This is calculated in the following proposition.

Proposition (2.5). (i) If $a$ and $b$ are natural numbers and $a_{k}=[k(a / b)]$ then

$$
\sum_{k=0}^{\infty} a_{k} t^{k}=\frac{\sum_{k=0}^{b-1} a_{k} t^{k}}{\left(1-t^{b}\right)}+\frac{a\left(\sum_{k=0}^{b-1} t^{k}\right) t^{b}}{\left(1-t^{b}\right)^{2}}
$$

(ii) The principal part of $P_{e}(t)$ at $t=1$ is

$$
\frac{e-1}{e} \frac{1}{(1-t)^{2}}-\frac{3(e-1)}{2 e} \frac{1}{(1-t)} \text {. }
$$

(iii) Suppose $\xi^{e}=1$ and $\xi \neq 1$. Then $P_{e}(t)$ has a pole of order 1 at $\xi$ with residue $\xi^{2} / e(1-\xi)$.

Proof. (i) Observe that $a_{k+n b}=a_{k}+n a$ and hence

$$
\begin{aligned}
\sum_{k=0}^{\infty} a_{k} t^{k} & =\sum_{k=0}^{b-1} \sum_{n=0}^{\infty} a_{k+n b} t^{k+n b}=\sum_{k=0}^{b-1} \sum_{n=0}^{\infty}\left(a_{k}+n a\right) t^{k+n b} \\
& =\sum_{k=0}^{b-1}\left(a_{k} t^{k} \sum_{n=0}^{\infty} t^{n b}+a \sum_{n=0}^{\infty} n t^{k+n b}\right) \\
& =\sum_{k=0}^{b-1}\left(a_{k} t^{k}\left(\frac{1}{1-t^{b}}\right)+a t^{k} \sum_{n=0}^{\infty} n t^{n b}\right) \\
& =\frac{\sum_{k=0}^{b-1} a_{k} t^{k}}{\left(1-t^{b}\right)}+\frac{a\left(\sum_{k=0}^{b-1} t^{k}\right)}{\left(1-t^{b}\right)^{2}} \cdot t^{b} .
\end{aligned}
$$

(ii) If we let $b=e$ and $a=e-1$ in part (i), then $a_{0}=a_{1}=0, a_{i}=i-1$ for $i=2, \ldots, e-1$. Let $z=1-t$ and substitute for $t$ in (2.5.1). We get

$$
P_{e}(t)=\frac{(e-2)(e-1) / 2}{e z}+\frac{(e-1) \sum_{k=0}^{e-1}(1-z)^{k}(1-z)^{e}}{e^{2} z^{2}}
$$

+ terms on nonnegative degree in $z$.

The principal part of the above is

$$
\frac{(e-1)(e-2)}{2 e}+\frac{(e-1) e}{e^{2} z^{2}}+\frac{e-1}{e^{2} z^{2}}\left[-e z-\sum_{k=1}^{e-1} k z\right]=\frac{(e-1)}{e z^{2}}-\frac{3(e-1)}{2 e z}
$$

(iii) $P_{e}(t)$ has a pole of order 1 at $t=\xi$, hence the residue $=\lim _{t \rightarrow \xi}(t-\xi) P_{e}(t)$ which can be evaluated using (2.5.1) and l'Hôpital's rule.

REMARK. Since $\mathscr{P}_{A}$ has a pole of order 2 at $t=1, A$ is an algebra of dimension 2 (cf. [A-M]). In particular this tells us that if $A$ is generated by $n$ elements, then the ideal of relations has at least $n-2$ generators.

Theorem (2.6). Suppose $G$ is a group with signature $\left\{g ; \sigma ; e_{1}, \ldots, e_{r}\right\}$. If the algebra of entire automorphic forms $A_{G}$ is generated by 3 elements $f_{0}, f_{1}, f_{2}$ of weights $q_{0}, q_{1}, q_{2}$ respectively, then the ideal of relations is a principal ideal generated by $a$ 
homogeneous element of degree $d$ and

$$
\begin{gathered}
2(g-1)+\sigma+\sum_{i=1}^{r} \frac{e_{i}-1}{e_{i}}=\frac{d}{q_{0} q_{1} q_{2}} \\
3(1-g)-\sigma-\frac{3}{2} \sum_{i=1}^{r} \frac{e_{i}-1}{e_{i}}=-\frac{d\left(d-q_{0}-q_{1}-q_{2}+2\right)}{2 q_{0} q_{1} q_{2}} \\
(1-g)-\frac{1}{2} \sum_{i=1}^{r} \frac{e_{i}-1}{e_{i}}=-\frac{d\left(d-q_{0}-q_{1}-q_{2}\right)}{2 q_{0} q_{1} q_{2}}
\end{gathered}
$$

Moreover

$$
\text { g.c.d. }\left(q_{0}, q_{1}, q_{2}\right)=1 \text {. }
$$

Proof. By (2.4) and Proposition (2.5) the principal part of $\mathscr{P}_{A}(t)$ at $t=1$ is

$$
\begin{aligned}
(2 g-2+\sigma & \left.+\sum_{i=1}^{r} \frac{e_{i}-1}{e_{i}}\right) \frac{1}{(1-t)^{2}} \\
& +\left(3-3 g-\sigma-\frac{3}{2} \sum_{i=1}^{r} \frac{e_{i}-1}{e_{i}}\right) \frac{1}{(1-t)}
\end{aligned}
$$

On the other hand we define $\varphi: \mathbf{C}\left[X_{0}, X_{1}, X_{2}\right] \rightarrow A$ by $\varphi\left(X_{i}\right)=f_{i}$, and we grade $S=\mathbf{C}\left[X_{0}, X_{1}, X_{2}\right]$ by letting degree $X_{i}=q_{i}$. We see that $A$ is isomorphic to $R=S / I$ as a graded algebra, where $I=\operatorname{kernel} \varphi$.

LEMMA (2.7). I is a principal ideal generated by a weighted homogeneous polynomial $F$ of some degree $d$.

We shall prove the lemma below. Returning to the proof of the theorem we apply Proposition (1.8) to find the principal part of $\mathcal{P}_{R}(t)$. Equating the coefficient of $1 /(1-t)^{2}$ in $\mathscr{P}_{R}(t)$ and $\mathscr{P}_{A}(t)$ gives (2.6.1).

Similarly equating the coefficient of $1 /(1-t)$ gives (2.6.2). The third equation follows from the first two. Finally g.c.d. $\left(q_{0}, q_{1}, q_{2}\right)=1$ since $\mathscr{P}_{A}(t)=\mathscr{P}_{R}(t)$ has a pole of order at most 1 at each nontrivial root of unity.

RemarK. The case that $A$ is generated by 2 elements is a special case of the above. If $f_{0}, f_{1}$ are generators of $A$ define $\varphi: S \rightarrow A$ by $\varphi\left(X_{i}\right)=f_{i}, i=0,1$, $\varphi\left(X_{2}\right)=0$. Grade $S$ by letting degree $X_{i}=q_{i}, i=0,1$, and degree $X_{2}=q_{2}$, arbitrary. Then $I$ is the principal ideal generated by $X_{2}$, so that $d=q_{2}$.

Proof of Lemma (2.7). If $\varphi: \mathrm{C}\left[X_{0}, X_{1}, X_{2}\right] \rightarrow A_{G}$ is a surjective graded homomorphism, then $I=\operatorname{kernel} \varphi$ is a principal ideal generated by a homogeneous element.

Proof. By (2.4) and Proposition (2.5), $\mathscr{P}_{A}(t)$ has a pole of order $\leqslant 2$ at $t=1$. By (2.1.1) the order of the pole is precisely 2 . Hence by (1.11)(b), $A$ has dimension 2. Now $A$ is isomorphic to $S / I$ hence $I$ is a height 1 prime ideal. But $S$ is a unique factorization domain, hence every height 1 prime ideal is principal $[\mathbf{Z}$, Chapter $\mathbf{V}$, §14]. If $F$ generates $I$ let $F=F_{d}+F_{d+1}+\cdots$ where $F_{i} \in S_{i}$. Now $I$ is a homogeneous ideal so $F_{d} \in I$. Thus $F \mid F_{d}$ and therefore $F=F_{d}$. 
Proposition (2.8). Suppose $G$ has signature $\left\{g ; \sigma ; e_{1}, \ldots, e_{r}\right\}$ and $A_{G}$ is generated by $f_{i} \in A_{q_{i}}, i=0,1,2$. For each $i$ let $\xi_{i}=\exp \left(2 \pi \sqrt{-1} / e_{i}\right)$ and $\nu_{i}=$ the number of $j$ so that $e_{i} \mid e_{j}$. Then

$$
\mathscr{P}_{A_{G}}(t)=\left(1-t^{d}\right) /\left(1-t^{q_{0}}\right)\left(1-t^{q_{1}}\right)\left(1-t^{q_{2}}\right)
$$

if and only if

(a) (2.6.1), (2.6.2) and (2.6.4) hold.

(b) For each $i$

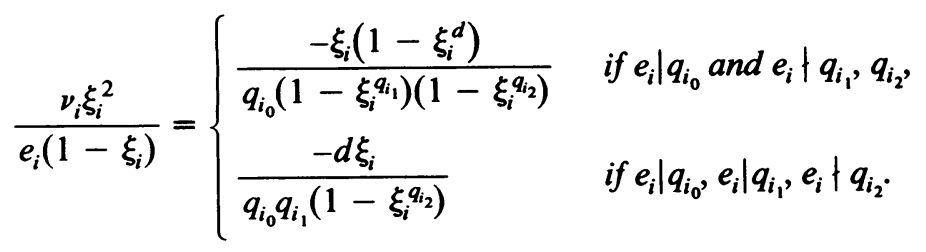

(c) For all $i$ and $j$ so that $i \neq j,\left(q_{i}, q_{j}\right) \mid d$.

(d)

$$
\begin{array}{ll}
d=q_{0}+q_{1}+q_{2}+1 & \text { if } \sigma=0, \\
d=q_{0}+q_{1}+q_{2} & \text { if } \sigma>0 \text { and } g>0, \\
d \leqslant q_{0}+q_{1}+q_{2}-1 & \text { if } \sigma>0 \text { and } g=0 .
\end{array}
$$

Proof. Let $f(t)=\left(1-t^{d}\right) /\left(1-t^{q_{0}}\right)\left(1-t^{q_{1}}\right)\left(1-t^{q_{2}}\right)$. Decompose $f(t)$ and $\mathscr{P}=$ $\mathcal{P}_{A_{G}}(t)$ as partial fractions over the complex numbers. The poles of $\mathscr{P}$ are at $e_{i}$ th roots of unity for some $e_{i}$ or at $t=1$. Equation (2.6.4) is equivalent to $f$ having a pole of order at most 1 at roots of unity. Equations (2.6.1) and (2.6.2) are equivalent to the coefficients of $1 /(1-t)$ and $1 /(1-t)^{2}$ being the same for $f$ and $\mathscr{P}$. By Propositions (2.5)(iii) and (1.9), equations (2.8.2) are equivalent to the equality of the principal parts of $f$ and $\mathscr{P}$ at $\xi$, where $\xi \neq 1$ is a root of unity. Now the polynomial part of $\mathscr{P}_{A}(t)$ is $\delta_{\sigma+1,1} t+g$. Thus $\sigma=0$ if and only if $\delta_{\sigma+1,1}=1$ if and only if $d=q_{0}+q_{1}+q_{2}+1$. If $\sigma>0$ and $g>0$ then the polynomial part of $\mathcal{P}$ is a nonzero constant, i.e., $\mathcal{P}$ is holomorphic and nonzero at $\infty$. But $f$ is holomorphic and nonzero at $\infty$ if and only if $d=q_{0}+q_{1}+q_{2}$. Finally $\sigma>0$ and $g=0$ is equivalent to $\mathscr{P}$ having a zero at $\infty$ and $f$ has a zero at $\infty$ if and only if $d \leqslant q_{0}+q_{1}+q_{2}-1$. When all other conditions are satisfied the constant term of $\mathscr{P}$ and $f$ must be equal since $f(0)=\mathscr{P}(0)=1$.

3. Groups for which $A_{G}$ may have few generators. Henceforth we assume $G$ is a finitely-generated Fuchsian group of the first kind with signature $\left\{g ; e_{1}, \ldots, e_{r}, \ldots, e_{r+\sigma}\right\}$ where $e_{i}<\infty$ for $1 \leqslant i \leqslant r$ and $e_{i}=\infty$ for $i>r$. We arrange the $e_{i}$ so that $e_{1} \leqslant e_{2} \leqslant \cdots \leqslant e_{r}$.

THEOREM (3.1). If the algebra $A_{G}$ is generated by 2 elements then it is a polynomial ring in two variables. Those signatures and the degrees $q_{0}, q_{1}$ of the generators are given below. 


$\begin{array}{cc}\text { Signature } & \left(q_{0}, q_{1}\right) \\ \{0 ; \infty, \infty, \infty\} & (1,1) \\ \{0 ; 2, \infty, \infty\} & (1,2) \\ \{0 ; 2,3, \infty\} & (2,3)\end{array}$

If the algebra $A_{G}$ is generated by 3 elements, then $G$ is on Table 1 which follows. For these signatures we determine the degrees $q_{0}, q_{1}, q_{2}$ of the generators and the degree $d$ of the relation. The converse of this theorem will be proven in \$4.

TABLE 1

\begin{tabular}{|c|c|c|c|}
\hline Signature & $\left(d ; q_{0}, q_{1}, q_{2}\right)$ & Signature & $\left(d ; q_{0}, q_{1}, q_{2}\right)$ \\
\hline [3], non hyperelliptic & $(4 ; 1,1,1)$ & $(0,2,2,2,2,3\}$ & $(8 ; 2,2,3)$ \\
\hline$\{2\}$ & $(6 ; 1,1,3)$ & $\{0 ; 2,2,2,2,2\}$ & $(10 ; 2,2,5)$ \\
\hline$\{2 ; 2\}$ & $(5 ; 1,1,2)$ & $(0 ; 2,3,3,3\}$ & $(9 ; 2,3,3)$ \\
\hline$\{1 ; 2,2,2\}$ & $(6 ; 1,2,2)$ & $\{0 ; 2,2,3,4\}$ & $(10,2,3,4)$ \\
\hline$\{1,2,2\}$ & $(8 ; 1,2,4)$ & $\{0 ; 2,2,3,3\}$ & $(12 ; 2,3,6)$ \\
\hline$\{1 ; 2,3\}$ & $(7 ; 1,2,3)$ & $\{0 ; 2,2,2,5\}$ & $(12 ; 2,4,5)$ \\
\hline$\{1 ; 2\}$ & $(12 ; 1,4,6)$ & $\{0 ; 2,2,2,4\}$ & $(14 ; 2,4,7)$ \\
\hline$\{1 ; 3\}$ & $(10 ; 1,3,5)$ & $\{0 ; 2,2,2,3\}$ & $(18 ; 2,6,9)$ \\
\hline$\{1 ; 4\}$ & $(9 ; 1,3,4)$ & $\{0 ; 4,4,4\}$ & $(12 ; 3,4,4)$ \\
\hline$(1 ; \infty, \infty, \infty)$ & $(3 ; 1,1,1)$ & $\{0,3,4,5\}$ & $(13 ; 3,4,5)$ \\
\hline$\{1 ; \infty, \infty\}$ & $(4 ; 1,1,2)$ & $\{0 ; 3,4,4\}$ & $(16 ; 3,4,8)$ \\
\hline$\{1 ; \infty)$ & $(6 ; 1,2,3)$ & $\{0 ; 3,3,4\}$ & $(24 ; 3,8,12)$ \\
\hline$\{0 ; \infty, \infty, \infty, \infty\}$ & $(2 ; 1,1,1)$ & $\{0 ; 3,3,5\}$ & $(18 ; 3,5,9)$ \\
\hline$(0 ; 2, \infty, \infty, \infty)$ & $(3 ; 1,1,2)$ & $\{0 ; 3,3,6\}$ & $(15 ; 3,5,6)$ \\
\hline$\{0 ; 2,2, \infty, \infty\}$ & $(4 ; 1,2,2)$ & $\{0 ; 2,5,6\}$ & $(16 ; 4,5,6)$ \\
\hline$\{0 ; 3, \infty, \infty\}$ & $(4 ; 1,2,3)$ & $\{0 ; 2,5,5\}$ & $(20 ; 4,5,10)$ \\
\hline$\{0 ; 2,2,2, \infty\}$ & $(6 ; 2,2,3)$ & $\{0 ; 2,4,7\}$ & $(18 ; 4,6,7)$ \\
\hline$\{0 ; 3,3, \infty)$ & $(6 ; 2,3,3)$ & $\{0 ; 2,4,6\}$ & $(22 ; 4,6,11)$ \\
\hline \multirow[t]{4}{*}{$0 ; 2,4, \infty$} & $(6 ; 2,3,4)$ & $\{0 ; 2,4,5\}$ & $(30 ; 4,10,15)$ \\
\hline & & $\{0 ; 2,3,9\}$ & $(24 ; 6,8,9)$ \\
\hline & & $\{0 ; 2,3,8\}$ & $(30 ; 6,8,15)$ \\
\hline & & $\{0 ; 2,3,7\}$ & $(42 ; 6,14,21)$ \\
\hline
\end{tabular}

Proof. For each possible signature we shall determine the degrees $q_{0}, q_{1}, q_{2}$ of the generators. Then $d$ is determined by (2.6.1). For clarity we shall divide the proof into sections.

(3.2) Suppose $A$ is generated by forms $f_{0}, f_{1}, f_{2}$ and $q_{i}=$ degree $f_{i}$. We let $S=\mathrm{C}\left[X_{0}, X_{1}, X_{2}\right]$ and define $\varphi: S \rightarrow A$ by $\varphi\left(X_{i}\right)=f_{i}$. We grade $S$ by letting degree $X_{i}=q_{i}$. Then $\varphi$ is onto and $A$ is isomorphic to $R=S / I$, where $I=\operatorname{kernel} \varphi$. Now by (2.3)

$$
\operatorname{dim} A_{1}= \begin{cases}(g-1)+\sigma & \text { if } \sigma>0 \\ g & \text { if } \sigma=0\end{cases}
$$

and $\operatorname{dim} A_{1} \leqslant 3$ since $A$ is generated by 3 elements. Thus

$$
(g-1)+\sigma \leqslant 3 \text { if } \sigma>0, \text { and } g \leqslant 3 \text { if } \sigma=0 .
$$

In either case $g \leqslant 3$. 
(3.3) Suppose $g=3$. Then by (3.2.1) either $\sigma=0$ or $\sigma=1$. In either case $\operatorname{dim} A_{1}=3$ implies there are at least 3 generators of weight 1 . Hence $q_{0}=q_{1}=q_{2}$ =1. Applying (2.3.1) we get $\operatorname{dim} A_{2}=6+2 \sigma+r$. But $\operatorname{dim} S_{2}=c(1,1,1)_{2}=6$ (see Definition (1.10)), hence, $\sigma=r=0$. Thus $\{3\}$ is the only possible signature with $g=3$. If $X$ is hyperelliptic, then the one-forms do not generate $A_{G}$ (this follows from [S, p. 293]).

(3.4) Now suppose $g=2$ or $g=1$. Then Table 2 gives $\operatorname{dim} A_{K}$ for all signatures which do not appear on Table 1. A generator of $A_{G}$ is indicated by the letters $a, b$, $c, d$. We stop counting generators after we have found that there must be four generators. As an example, consider the signature $\left\{1 ; 2, e_{2}\right\}, e_{2}>3$. Clearly there is a generator $a \in A_{1}$. Now $\operatorname{dim} A_{2}=2$, so there must be an element $b \in A_{2}$ so that $a^{2}$ and $b$ are linearly independent. Next there must be an element $c \in A_{3}$ so that $a^{3}, a b$ and $c$ are independent. Finally, $a, b$ and $c$ generate a subspace of dimension $\leqslant c(1,2,3)_{4}=4$ in $A_{4}$, so there must be a fourth generator $d$.

TABLE 2. $\operatorname{dim} A_{k}$ for signatures with embedding dimension

\begin{tabular}{|c|c|c|c|c|c|c|}
\hline & $k$ & 1 & 2 & 3 & 4 & 5 \\
\hline$\left\{2 ; a_{1}, \ldots, e_{r}\right\}$ & $r \geq 2$ & 2,0 & $\begin{array}{l}c, d \\
3+r\end{array}$ & & & \\
\hline$\left\{2 ; e_{1}\right\}$ & $e_{1}>2$ & $2, \dot{0}$ & $\begin{array}{l}c \\
4\end{array}$ & $\begin{array}{l}\mathrm{d} \\
7\end{array}$ & & \\
\hline$\left\{2 ; c ; e_{1}, \ldots, e_{r}\right\}$ & $\sigma \geq 3$ & $a, \underset{\leq 4}{a, c, d}$ & & & & \\
\hline$\left\{2 ; \sigma ; e_{1}, \ldots, e_{r}\right\}$ & $\sigma=2$ & $\underset{3}{a, b}, c$ & $\geq \frac{d}{7}$ & & & \\
\hline$\left\{2 ; c ; 2_{1}, \ldots, 2_{r}\right\}$ & $\sigma=1$ & $a_{2}^{a, b}$ & $\begin{array}{l}c, d \\
\geq 5\end{array}$ & & & \\
\hline$\left\{1 ; e_{1}, \ldots, e_{r}\right\}$ & $r \geq 3$ & $\begin{array}{l}a \\
1\end{array}$ & $\underset{r}{b, c, d}$ & & & \\
\hline$\left\{1 ; e_{1}, e_{2}, e_{3}\right\}$ & $e_{3}>2$ & $\begin{array}{l}a \\
i\end{array}$ & $\mathrm{~b}, \mathrm{c}_{3}$ & $\begin{array}{r}d \\
\geq 4\end{array}$ & & \\
\hline$\left\{1 ; e_{1}, e_{2}\right\}$ & $e_{1}>2$ & $\begin{array}{l}a \\
I\end{array}$ & $\begin{array}{l}b \\
2\end{array}$ & $c, d$ & & \\
\hline$\left\{1 ; 2 ; e_{2}\right\}$ & $e_{2}>3$ & $\begin{array}{l}a \\
1\end{array}$ & $\begin{array}{l}\mathrm{b} \\
2\end{array}$ & $\begin{array}{l}c \\
3\end{array}$ & $\begin{array}{l}d \\
5\end{array}$ & \\
\hline$\left\{1 ; e_{1}\right\}$ & $e_{1}>4$ & a & 1 & $\begin{array}{l}b \\
2\end{array}$ & $\begin{array}{l}c \\
3\end{array}$ & $\begin{array}{l}d \\
4\end{array}$ \\
\hline$\left(1 ; c ; e_{1}, \ldots, e_{r}\right\}$ & $\sigma>3$ & $a, b, c, d$ & & & & \\
\hline$\left\{1 ; 3 ; e_{1}, \ldots, e_{r}\right\}$ & $r>0$ & $\underset{3}{a, b}, c$ & $\begin{array}{r}d \\
\geq 7\end{array}$ & & & \\
\hline$\left\{1 ; 2 ; e_{1}, \ldots, e_{r}\right\}$ & $r>0$ & $\frac{a, b}{2}$ & $\begin{array}{l}c, d \\
\geq 5\end{array}$ & & & \\
\hline$\left\{1 ; e_{1} ; \infty\right\}$ & & à & ${ }_{3}^{b, c}$ & $\geq 4$ & & \\
\hline$\left\{0 ; \sigma ; e_{1}, \ldots, e_{r}\right\}$ & $\sigma>4$ & $\begin{array}{l}a, b, c, d \\
\sigma-1\end{array}$ & & & & \\
\hline$\left\{0 ; 4 ; e_{1}, \ldots, e_{r}\right\}$ & & $\underset{3}{a, b, c}$ & $r+5$ & 7 & & \\
\hline
\end{tabular}


(3.5) Suppose $g=0$. Then Table 3 lists all the groups with signatures not on Table 1. This table suffices to rule out all the cases except $\sigma=2, r=1, e_{1}>4$. In that case $q_{0}=1, q_{1}=2$ and $q_{2}=3$. By $(2.6 .1), d / 6=\left(e_{1}-1\right) / e_{1}$ which implies $e_{1}=3$ or 6 . But $e_{1}=6$ does not satisfy (2.6.3). Thus $e_{1}=3$.

$\{0 ; 2,2, \infty\}$ is not realizable by $(2.1 .1)$.

4. Classification. In this section we prove the converse of Theorem (3.1); for every group $G$ on Table $1, A_{G}$ is generated by 3 or 2 elements. In order to do this, we first reinterpret the ring $A_{G}$ as a ring of functions on $X$.

(4.1) Let $\omega^{*}$ be an arbitrary meromorphic differential form on $\bar{X}$ and $K$ the divisor of $\omega^{*}$. Then $\omega^{*}$ pulls back to a meromorphic form $\omega$ on $H_{+}$. If $f$ is an automorphic form of weight $k, g=f / \omega^{k}$ is a $G$-invariant meromorphic function on $H_{+}$and hence induces a meromorphic function on $\bar{X}$. By [G, p. 24] $f$ is holomorphic if and only if

$$
(g)+k \cdot K+\sum_{i=1}^{r}\left[k\left(1-\frac{1}{e_{i}}\right)\right] p_{i}+\sum_{r+1}^{r+\sigma} k p_{i}>0
$$

where $p_{1}, \ldots, p_{r}$ are the elliptic points and $p_{r+1}, \ldots, p_{r+\sigma}$ are the cusp points. Here ( ) denotes the divisor of a function or differential. Thus for any $k$ we have an isomorphism

$$
\varphi_{k}: A_{k} \stackrel{\approx}{\rightarrow} L\left(k \cdot K+\sum_{i=1}^{r}\left[k\left(1-\frac{1}{e_{i}}\right)\right] p_{i}+\sum_{i=1}^{\sigma} k p_{r+i}\right)
$$

TABLE 3. $\operatorname{dim} A_{k}$ for signatures with embedding dimension

$$
>3, g=0, \sigma \leqslant 2
$$
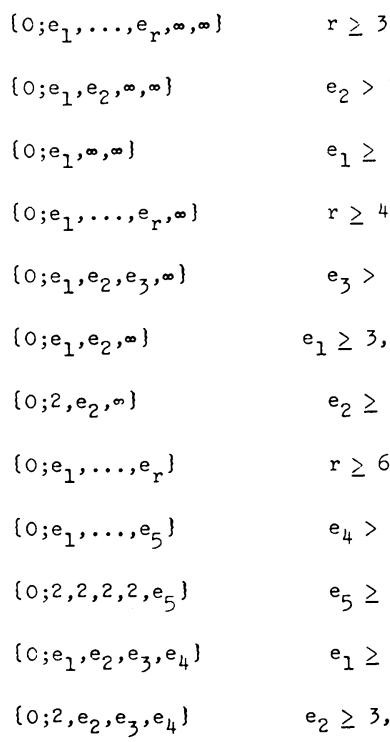

$e_{2}>2$

$e_{1} \geq 4$

$r \geq 4$

$e_{3}>2$

$e_{1} \geq 3, e_{2} \geq 40$

$e_{2} \geq 5$

$r \geq 6$

$e_{4}>2$

$e_{5} \geq 4$

$e_{1} \geq 3$

$e_{2} \geq 3, e_{4}>30$

$\begin{array}{cc}2 & 3 \\ b, c, d & \\ i+r, c & d \\ 3 & \geq \\ b & c \\ 2 & 3 \\ a, b, c & d \\ r-1 & \geq \\ a, b & c, d \\ 2 & \geq \\ a & b \\ 1 & 2 \\ a & b \\ 1 & 1 \\ a, b, c & d \\ r-3 & \geq \\ a, b & c \\ 2 & \geq \\ a, b & c \\ 2 & 1 \\ a & b, c \\ 1 & 3 \\ a & b, c \\ 1 & 2\end{array}$

$\geq 2$

$c$
2

$\frac{d}{2}$

$\geq 1$

$c, d$

$\begin{array}{ll}c & d \\ 1 & 4\end{array}$

${ }_{3}, d$

$b^{b}, \quad d 2$ 


\section{TABLE 3 (continued)}

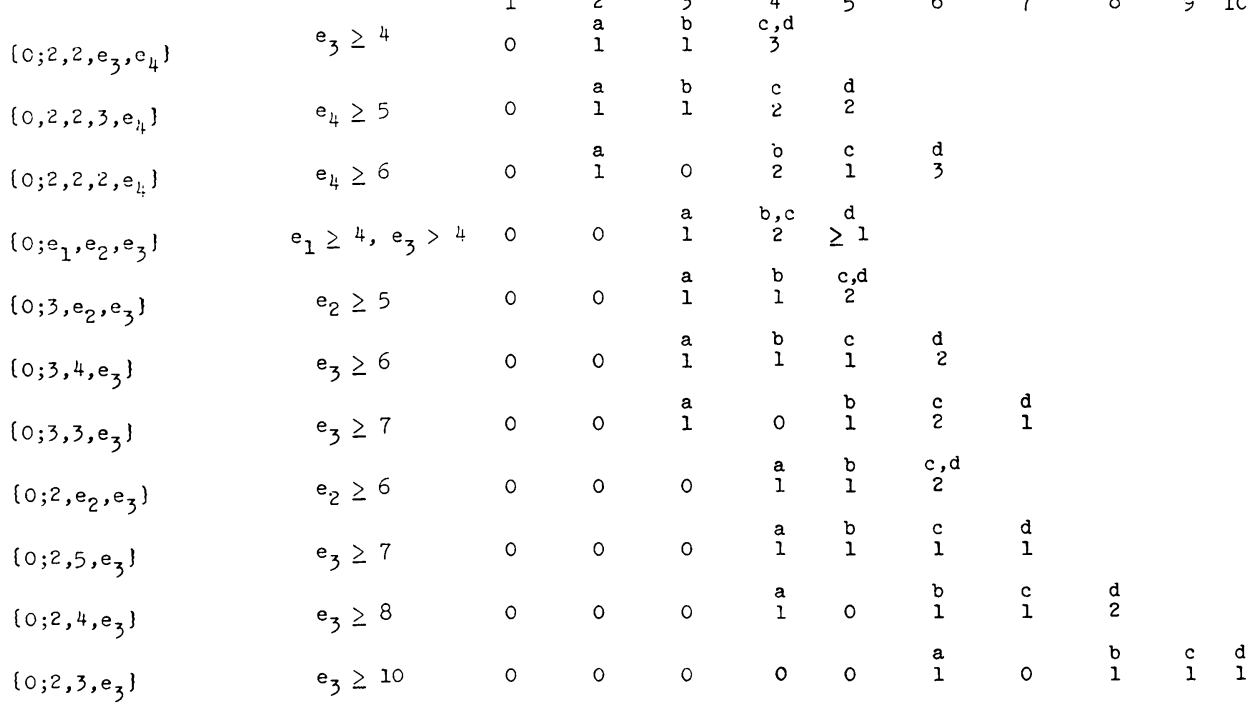

defined by $\varphi_{k}(f)=f / \omega^{k}$. Let

$$
L_{k}=L\left(k \cdot K+\sum_{i=1}^{r}\left[k\left(1-\frac{1}{e_{i}}\right)\right] p_{i}+\sum_{i=1}^{\sigma} k p_{r+i}\right) .
$$

Then we have an isomorphism of $\mathbf{C}$-algebras

$$
\varphi: A_{G} \stackrel{\approx}{\rightarrow} \underset{k>0}{\bigoplus} L_{k}
$$

Let $L_{G}$ denote $\bigoplus_{k>0} L_{k}$.

REMARK. $\varphi_{k}$ induces an isomorphism of the space of cusp forms of weight $k$ with a similar vector space of functions

$$
\varphi_{k}: C_{k} \underset{\rightarrow}{\approx} L\left(k \cdot K+\sum_{i=1}^{r}\left[k\left(1-\frac{1}{e_{i}}\right)\right] p_{i}+\sum_{i=1}^{\sigma}(k-1) p_{r+i}\right) .
$$

One can use this to see that the algebra of cusp forms is not finitely generated, as follows. Linear combinations of products of cusp forms of degree $<k$ are all contained in

$$
\bar{C}_{k}=L\left(k \cdot K+\sum_{i=1}^{r}\left[k\left(1-\frac{1}{e_{i}}\right)\right] p_{i}+\sum_{i=1}^{\sigma}(k-2) p_{r+i}\right) .
$$

If $g \geqslant 1$, it follows immediately from Riemann-Roch that $\operatorname{dim} C_{k} / \bar{C}_{k} \geqslant \sigma$, and hence that there are at least $\sigma$ generators of every degree. If $g=0$ then we have $A_{k} \supset C_{k} \supset \bar{C}_{k}$ and again by Riemann-Roch $\operatorname{dim} C_{k} / \bar{C}_{k}>0$, provided $\operatorname{dim} A_{k}>$ o. Now $A$ is an algebra of dimension 2 (see Remark preceding Theorem (2.6)) hence there are an infinite number of $k$ so that $\operatorname{dim} A_{k} \geqslant \sigma$. Thus $C_{k}$ requires an infinite number of generators. 
(4.2) Let $\mathbf{C}(X)$ denote the field of meromorphic functions on $X$. Note that the inclusions $L_{k} \rightarrow \mathrm{C}(X)$ do not induce an inclusion $L_{G} \rightarrow \mathbf{C}(X)$. (Since, for example, $L_{k} \subset L_{2 k}$.) Let $t$ be an indeterminate and define

$$
L_{k} \rightarrow \mathbf{C}(X)[t], \quad f \mapsto f \cdot t^{k} .
$$

This extends to an injective $\operatorname{map} i_{G}: L_{G} \rightarrow \mathbf{C}(X)[t]$.

Henceforth, we identify $L_{G}$ with its image in $\mathbf{C}(X)[t]$, i.e., an element $(0, \ldots, 0, f, 0, \ldots)$ is identified with $f \cdot t^{k}$ if $f$ lies in the $k$ th component. Thus the exponent of $t$ serves as a reminder of which $L_{k} f$ comes from.

ExAmple (4.3). Suppose $G$ is a group with signature $\{1 ; 4\}$. Then $L_{k} \approx L\left(\left[k \frac{3}{4}\right] p\right)$ where $p \in X$ is the branch point. Let $f$ be the Weierstrass $\wp$ function with pole (of order 2) at $p$ and $g=f^{\prime}$. Then letting $\langle>$ denote "vector space generated by", we have

$$
L(n p)= \begin{cases}\langle 1\rangle, & n=0,1, \\ \left\langle 1, f, g, f^{2}, f^{2} g, \ldots, f^{n / 2}\right\rangle, & n \text { even, } \geqslant 2, \\ \left\langle 1, f, g, f^{2}, f^{2} g, f^{3}, \ldots, f^{n-3 / 2} g\right\rangle, & n \text { odd, } \geqslant 3 .\end{cases}
$$

Now

$$
L_{k}= \begin{cases}L(3 n p), & k=4 n \\ L(3 n p), & k=4 n+1 \\ L((3 n+1) p), & k=4 n+2 \\ L((3 n+2) p), & k=4 n+3\end{cases}
$$

$L_{G}=\langle 1\rangle \oplus\langle 1\rangle t \oplus\langle 1\rangle t^{2} \oplus\langle 1, f\rangle t^{3} \oplus\langle 1, f, g\rangle t^{4} \oplus\langle 1, f, g\rangle t^{5} \ldots$. It can easily be verified that $L_{G}$ is generated by $z_{0}=1 \cdot t, z_{1}=f \cdot t^{3}$ and $z_{2}=g \cdot t^{4}$. The map

$$
\Phi: \mathbf{C}\left[Z_{0}, Z_{1}, Z_{2}\right] \rightarrow L_{G}
$$

defined by $\Phi\left(Z_{i}\right)=z_{i}$ is surjective. By Lemma (2.7) the kernel of $\Phi$ is a principal ideal generated by a homogeneous polynomial $f$ of degree $d$. Now by Proposition (2.8)(d), $d=q_{0}+q_{1}+q_{2}+1=9$ and we see that

$$
f\left(Z_{0}, Z_{1}, Z_{2}\right)=Z_{0} Z_{2}^{2}-4 Z_{1}^{3}+g_{2} Z_{0}^{6} Z_{1}+g_{3} Z_{0}^{9}
$$

where $g_{2}$ and $g_{3}$ are constants depending on $G$ so that $g_{2}^{3}-27 g_{3}^{2} \neq 0$.

For the groups on Table 1 we have natural candidates for generators of $A_{G}$. To show that these elements actually generate we use the following.

Proposition (4.4). Suppose $A$ is a graded algebra over C,

$$
\mathscr{P}_{A}(t)=\frac{1-t^{d}}{\left(1-t^{q_{0}}\right)\left(1-t^{q_{1}}\right)\left(1-t^{q_{2}}\right)}, \quad z_{i} \in A_{q_{i}} \text { for each } i=0,1,2,
$$

and the $z_{i}$ satisfy a relation $f$ of degree $d$ in $A$. Suppose, moreover, that

(1) $f$ is irreducible,

(2) the $z_{i}$ generate a field $\mathbf{C}\left(z_{0}, z_{1}, z_{2}\right)$ of transcendence degree 2.

Then the canonical map

$$
\Phi: \mathbf{C}\left[Z_{0}, Z_{1}, Z_{2}\right] /(f) \rightarrow A
$$

is an isomorphism. 
Proof. Let $R=\mathrm{C}\left[Z_{0}, Z_{1}, Z_{2}\right] /(f)$ and $I=\operatorname{kernel} \Phi$. Now $f$ irreducible implies $R$ is an integral domain. Thus $I \neq(0)$ implies [A-M, $\S 11] \operatorname{dim} R / I<\operatorname{dim} R=2$. But transcendence degree $\mathbf{C}\left(z_{0}, z_{1}, z_{2}\right)=2$ implies $\operatorname{dim} R / I=2$ [A-M, 11.25]. Thus $I=(0)$ and hence $\Phi$ is one-one. Finally, $\mathscr{P}_{R}(t)=\mathscr{P}_{A}(t)$ implies $\Phi$ is onto.

Remark (4.5). Suppose $A$ is an integral domain, $A, \mathscr{P}_{A}$ and $z_{i}$ are as in Proposition (4.4) and the $z_{i}$ satisfy a relation $f$ of degree $d$.

(1) If the $z_{i}$ satisfy no relation of degree $<d$ then $f$ is irreducible.

(2) If for some $i \neq j, z_{i}^{q_{j}} / z_{j}^{q_{i}}$ is not a constant then the $z_{i}$ generate a field $\mathbf{C}\left(z_{0}, z_{1}, z_{2}\right)$ of transcendence degree 2 .

Proof. (1) If $f$ were reducible then $f=g h$ where $g$ and $h$ are homogeneous of degree $<d$. But $A$ is an integral domain and hence either $g$ or $h$ is a relation in $A$. This contradicts the hypothesis.

(2) If $\mathbf{C}\left(z_{0}, z_{1}, z_{2}\right)$ has transcendence degree $\leqslant 1$, then $z_{0}$ and $z_{1}$ are algebraically dependent. Then there is an irreducible polynomial $f\left(Z_{0}, Z_{1}\right)$ satisfied by $z_{0}$ and $z_{1}$. But $f$ is weighted homogeneous of the form $Z_{0}^{\alpha}-\varepsilon Z_{1}^{\beta}$ where $\varepsilon \in \mathbf{C}, \alpha=$ $q_{1} /\left(q_{0}, q_{1}\right)$ and $\beta=q_{0} /\left(q_{0}, q_{1}\right)$ by [O-W, Lemma 3.6]. Thus $z_{0}^{q_{1}} / z_{1}^{q_{0}}$ is a power of $\varepsilon$, contradicting the hypothesis.

Theorem (4.6). For every group on Table 1 in $\$ 3, A_{G}$ is generated by $\leqslant 3$ elements. The degrees of the generators and the relation are given in Table 1.

Proof. For each group one finds natural candidates, $z_{i} \in L_{q_{i}} \cdot t^{q_{i}}, i=0,1,2$, for the generators of $L_{G}$ using Table 4 . Then we show that these actually generate using Proposition (4.4) and Remark (4.5). One first verifies that for each group $G$

$$
\mathscr{P}_{A^{(t)}}=\left(1-t^{d}\right) /\left(1-t^{q_{0}}\right)\left(1-t^{q_{1}}\right)\left(1-t^{q_{2}}\right)
$$

where $q_{0}, q_{1}, q_{2}$ and $d$ are given in Table 1 . This verification can be carried out using Proposition (2.8) or (2.4) and Proposition (2.5) and we shall not include these calculations here. The fact that the $z_{i}$ satisfy a relation $f$ of degree $d$ is also easily seen. Hence all that remains is to show that Proposition (4.4)(1) or Remark (4.5)(1) and Proposition (4.4)(2) or Remark (4.5)(2) are satisfied. We first prove a technical lemma which is used in the proof.

LEMMA (4.6.1). Suppose $f_{0}, \ldots, f_{r}$ are analytic functions, none of which is identically zero. Suppose that for each $i$ there is a point $p$ so that $\nu_{p}\left(f_{i}\right)<\nu_{p}\left(f_{j}\right)$ for all $i<j$, where $\nu_{p}(f)$ is the order of the pole of $f$ at $p$. Then $f_{0}, \ldots, f_{r}$ are linearly independent.

Proof. Proof by induction. The assertion is true for $r=0$. Now suppose it is true for $r-1$ functions. If $f_{0}, \ldots, f_{r}$ are as before and $a_{0} f_{1}+\cdots+a_{r-1} f_{r-1}+$ $a_{r} f_{r}=0$ then $a_{0} f_{0}+\cdots+a_{r-1} f_{r-1}=-a_{r} f_{r}$. If $a_{r} \neq 0$, then by hypothesis there is a point $p$ so that the right-hand side has a pole at $p$ of order, say $n=\nu_{p}\left(f_{r}\right)$, while the left-hand side has a pole of lesser order. This is impossible, so $a_{r}=0$. Now by the inductive hypothesis $f_{0}, \ldots, f_{r-1}$ are linearly independent so $a_{0}=a_{1}=\cdots=$ $a_{r-1}=0$. Thus $f_{0}, \ldots, f_{r}$ are linearly independent.

We return to the proof of the theorem, examining each signature in turn. 


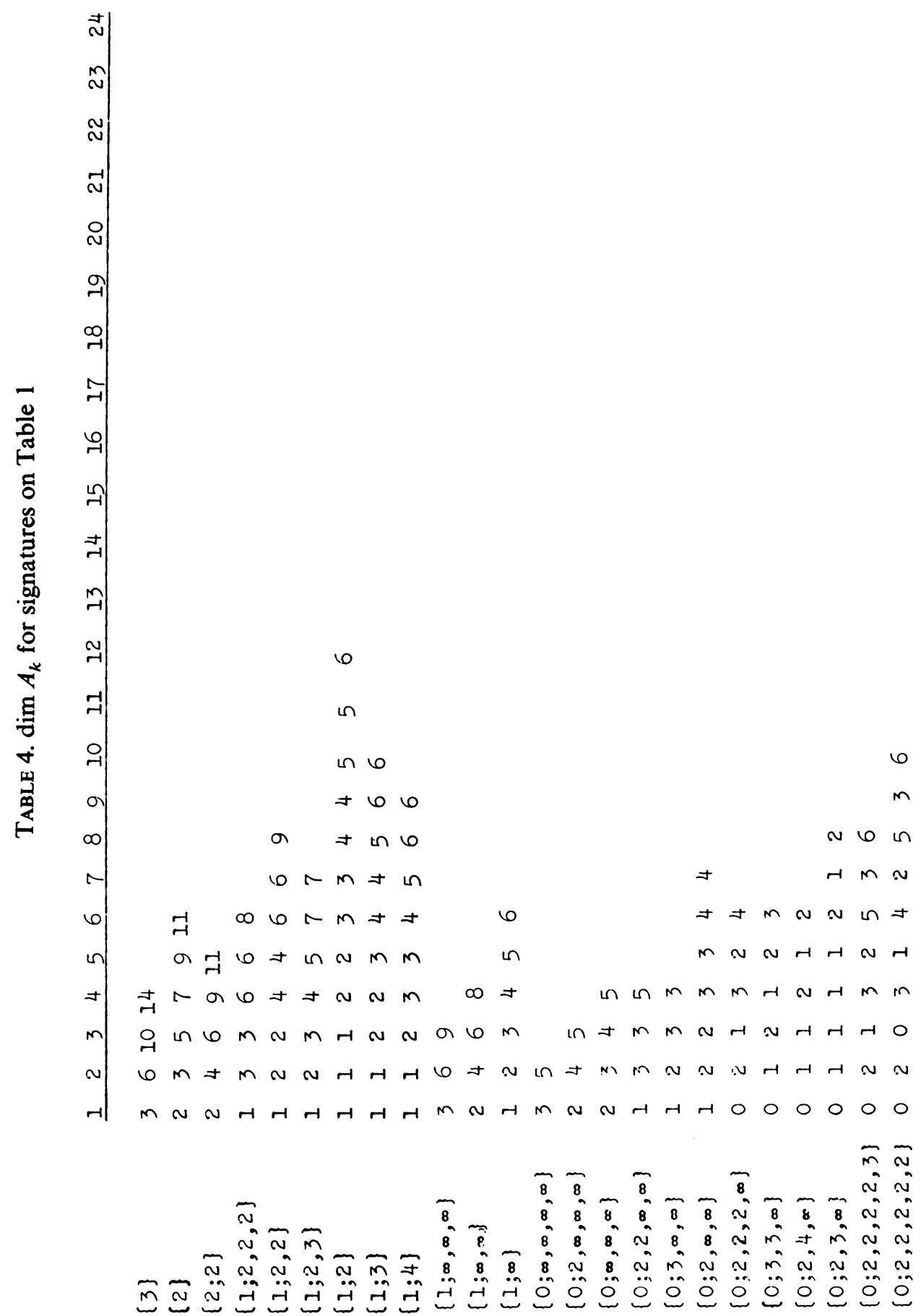




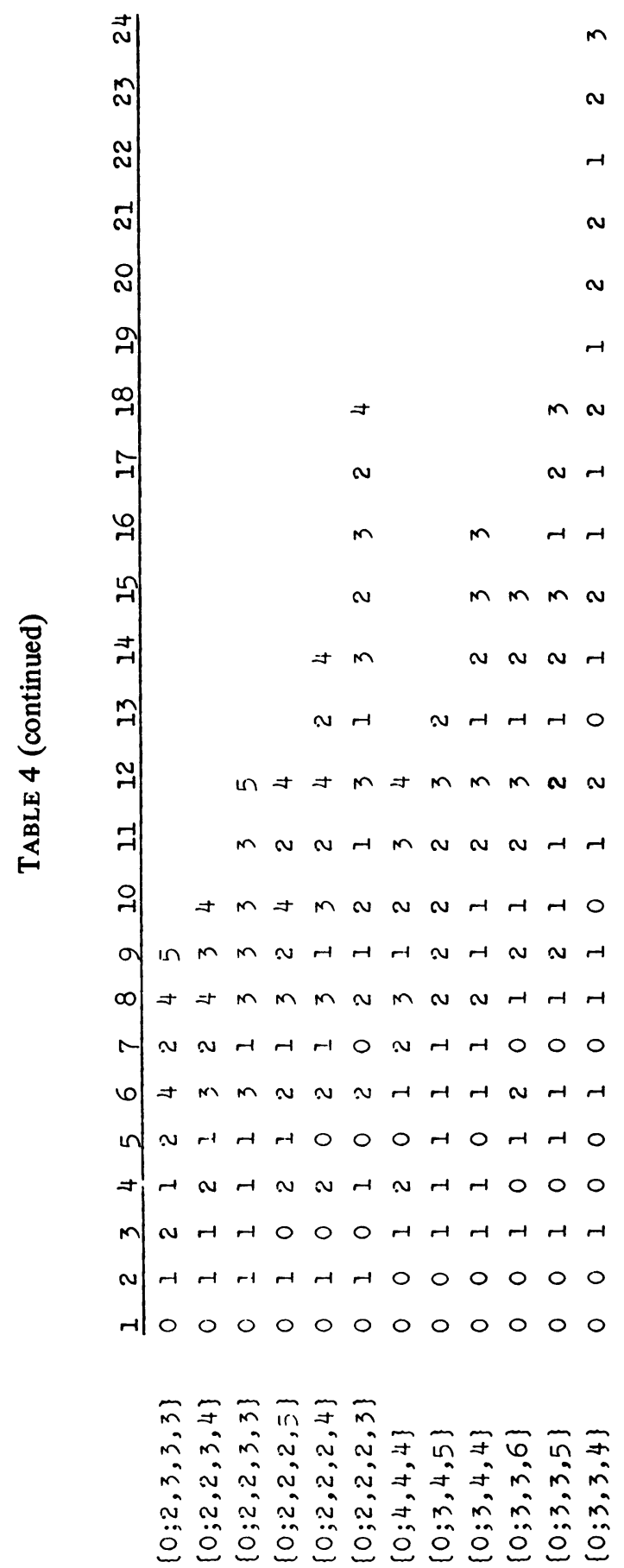




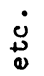

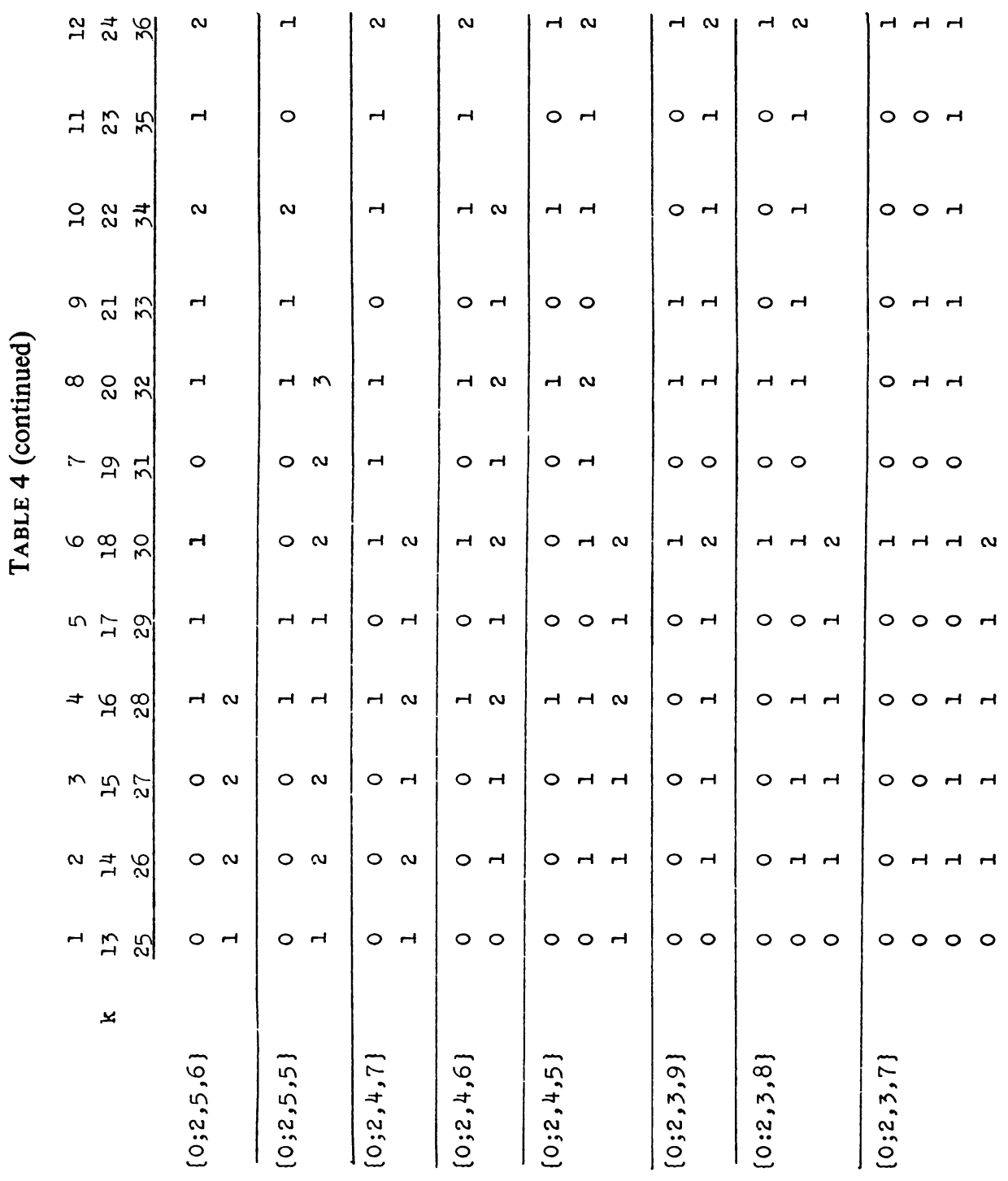


\{3\}: By Noether's theorem [S-D] $A_{G}$ is generated by 1 -forms if and only if $X$ is nonhyperelliptic. In fact, we can see this rather explicitly in the case of genus 3 . If $X$ is nonhyperelliptic then one can choose a basis $\omega_{1}, \omega_{2}, \omega_{3}$ for the space of 1 -forms and then

$$
h(x)=\left(\omega_{1}(x): \omega_{2}(x): \omega_{3}(x)\right)
$$

defines the canonical embedding of $X$ in $P^{2}$. Now one can see that $A_{G}$ is isomorphic to the homogeneous coordinate ring of $h(X)$. Hence $A_{G}$ is generated by the $\omega_{i}$, and the $\omega_{i}$ satisfy a (nonsingular) relation of degree 4. This relation is precisely the equation of $h(X)$ in $P^{2}$. Conversely, if $X$ is hyperelliptic we can write $X$ as the Riemann surface of the plane curve

$$
y^{2}=\prod_{i=1}^{7}\left(x-\varepsilon_{i}\right)
$$

By $[\mathbf{S}, \S 10.10]$ the 1-forms are generated by $d x / y, x d x / y, x^{2} d x / y$ and the 2-forms by $d x^{2} / y^{2}, \ldots, x^{4} d x^{2} / y^{2}, d x^{2} / y$. Now the last 2 -form is not in the subspace generated by products of 1 -forms, hence $A$ is not generated by 1 -forms. One can show that $A$ is generated by the three 1 -forms and $d x^{2} / y$.

\{2\}: We may write $X$ as the Riemann surface of the curve

$$
y^{2}=\left(x-\varepsilon_{1}\right)\left(x-\varepsilon_{2}\right)\left(x-\varepsilon_{3}\right)\left(x-\varepsilon_{4}\right)\left(x-\varepsilon_{5}\right)
$$

where the $\varepsilon_{i}$ are distinct nonzero complex numbers.

$$
\begin{aligned}
& A_{1}=\langle d x / y, x d x / y\rangle, \\
& A_{2}=\left\langle d x^{2} / y^{2}, x d x^{2} / y^{2}, x^{2} d x^{2} / y^{2}\right\rangle, \\
& A_{3}=\left\langle d x^{3} / y^{3}, x d x^{3} / y^{3}, x^{2} d x^{3} / y^{3}, x^{3} d x^{3} / y^{3}, d x^{3} / y^{2}\right\rangle, \\
& L_{1}=\langle 1, x\rangle, \quad L_{2}=\left\langle 1, x, x^{2}\right\rangle, \quad L_{3}=\left\langle 1, x, x^{2}, x^{3}, y\right\rangle .
\end{aligned}
$$

Let $z_{0}=1 \cdot t, z_{1}=x \cdot t, z_{2}=y \cdot t^{3}$. Then the $z_{i}$ generate a field of transcendence degree 2 and satisfy an irreducible polynomial relation

$$
\left(z_{2}\right)^{2}-z_{0} \prod_{i=1}^{5}\left(z_{1}-\varepsilon_{i} z_{0}\right)=0 .
$$

Thus by Proposition (4.4) the $z_{i}$ generate $L_{G}$.

$\{2 ; 2\}$ : The curve $X$ is given by equation (4.6.1). Let $p$ be the branch point on $X$. Suppose $p=\infty$. Then $L_{1}=L(K)=\langle 1, x\rangle, \quad L_{2}=L(2 K+p)=L(5 \infty)=$ $\left\langle 1, x, x^{2}, y\right\rangle$. Let $z_{0}=1 \cdot t, z_{1}=x \cdot t, z_{2}=y \cdot t^{2}$. Then the $z_{i}$ satisfy the irreducible relation

$$
z_{0} z_{2}^{2}-\prod_{i=1}^{5}\left(z_{1}-\varepsilon_{i} z_{0}\right)=0 .
$$

Hence by Proposition (4.4) the $z_{i}$ generate $L_{G}$.

Now suppose $p \neq \infty$. Then $L_{1}=\langle 1, x\rangle, L_{2}=\left\langle 1, x, x^{2}, f\right\rangle$ where $f$ has a pole of order 1 at $p$. By subtracting a suitable linear combination of $x$ and $x^{2}$ from $f$ we may replace $f$ by a function with a pole of odd order at infinity (since the canonical divisor $K=2 \infty)$. By the Riemann-Roch theorem $l(\infty+p)=1+l(\infty-p)=1$, 
hence $L(\infty+p)$ consists only of constants. Therefore $f$ must have a pole of order 3 at $\infty$. Now $L_{3} \supseteq\left\langle 1, x, x^{2}, x^{3}, f, x f\right\rangle$ and by using Lemma (4.6.1) we can show that those six functions are linearly independent. The dimension of $L_{3}$ is 6 , hence the inclusion above is an equality. Similarly $L_{4}=\left\langle 1, x, x^{2}, x^{3}, x^{4}, f, x f, x^{2} f, f^{2}\right\rangle$ and these functions are linearly independent. We can now exhibit candidates for generators of $L$, namely let $z_{0}=1 \cdot t, z_{1}=x \cdot t, z_{2}=f \cdot t^{2}$. One can easily verify that there are 12 monomials of (weighted) degree 5 in $z_{0}, z_{1}, z_{2}$ and that $\operatorname{dim} L_{5}=$ 11. Thus there must be a relation of degree 5 among the $z_{i}$. Moreover one can easily see from the preceding remarks that there is no relation of degree less than 5 . Hence by Remark (4.5) and Proposition (4.4) the $z_{i}$ generate $L$ and satisfy a relation of degree 5 .

$\{1 ; 2,2,2\}:$ In the case $g=1$, the canonical divisor $K=0$. Thus $L_{1}=L(0)=$ $\langle 1\rangle, L_{2}=L\left(p_{1}+p_{2}+p_{3}\right)=\langle 1, f, g\rangle$ where we choose $f$ and $g$ so that the divisor of poles of $f,(f)_{\infty}=p_{1}+p_{2}$ and $(g)_{\infty}=p_{2}+p_{3}$. Let $z_{0}=1 \cdot t, z_{1}=f \cdot t^{2}, z_{2}=g$ $\cdot t^{2}$. There is a relation of degree 6 among the $z_{i}$ since $\operatorname{dim} A_{6}=9$, while there are 10 monomials of degree 6 in the $z_{i}$. Now by Remark (4.5)(1) it is sufficient to show that there is no relation of degree $<6$. Now $L_{2}=L_{3}$ so there is no relation of degree 3. Next we see that

$$
L_{4}=L\left(2 p_{1}+2 p_{2}+2 p_{3}\right) \supseteq\left\langle 1, f, g, f^{2}, f g, g^{2}\right\rangle
$$

and $\operatorname{dim} L_{4}=6$. By considering the orders of the poles of the above functions at the $p_{i}$ we can see that they are linearly independent. Thus we have equality in (4.6.4) and there can be no relation of degree 4. Finally $L_{4}=L_{5}$ so there is no relation of degree 5 .

$\{1 ; 2,2\}:$ In this case $L_{1}=L(0)=\langle 1\rangle, L_{2}=L\left(p_{1}+p_{2}\right)=\langle 1, f\rangle, L_{3}=L_{2}$, $L_{5}=L_{4}=L\left(2 p_{1}+2 p_{2}\right)=\langle 1, f, g\rangle$ where we can choose $f$ and $g$ so that $(f)_{\infty}=$ $p_{1}+p_{2}$ and $(g)_{\infty}=2 p_{1}$. Then one can easily verify that $L_{6}=L_{7}=$ $\left\langle 1, f, f^{2}, f^{3}, g, f g\right\rangle$ since these functions are linearly independent. There is a relation of degree 8 among the $z_{i}$ since $\operatorname{dim} A_{8}=8$ and there are 9 monomials of degree 9 in the $z_{i}$. Now by the above remarks if we let $z_{0}=1 \cdot t, z_{1}=f \cdot t^{2}, z_{2}=g \cdot t^{4}$ then there are no relations of degree less than 8 , hence by Proposition (4.4) and Remark (4.5) the $z_{i}$ generate $L_{G}$.

$\{1 ; 2,3\}$ : In this case $L_{1}=\langle 1\rangle, L_{2}=\langle 1, f\rangle, L_{3}=\langle 1, f, g\rangle$ where $(f)_{\infty}=p_{0}+$ $p_{1}$ and $(g)_{\infty}=2 p_{1}$. Then one can easily verify that a basis for $L_{i}, i=4,5,6$, is given as follows.

$$
\begin{aligned}
& L_{4}=\left\langle 1, f, g, f^{2}\right\rangle, \quad L_{5}=\left\langle 1, f, g, f^{2}, f g\right\rangle, \\
& L_{6}=\left\langle 1, f, g, f^{2}, f g, g^{2}, f^{3}\right\rangle .
\end{aligned}
$$

Letting $z_{0}=1 \cdot t, z_{1}=f \cdot t^{2}, z_{2}=g \cdot t^{3}$ we see that there is a relation of degree 7 and no relation of degree $<7$. Hence again by Proposition (4.4) and Remark (4.5) the $z_{i}$ generate $L_{G}$.

$\{1 ; n\}$ : Let $p$ be the branch point, $\wp$ the Weierstrass function with pole at $p$. Let $f=\wp$ and $g=\wp$ and $z_{0}=1 \cdot t$. If $n=2$, let $z_{1}=f \cdot t^{4} \in L_{4} \cdot t^{4}, z_{2}=g \cdot t^{6} \in L_{6}$. $t^{6}$. If $n=3$, let $z_{1}=f \cdot t^{3} \in L_{3} \cdot t^{3}$ and $z_{2}=g \cdot t^{5} \in L_{5} \cdot t^{5}$. If $n=4$, let $z_{1}=f \cdot t^{3}$ $\in L_{3} \cdot t^{3}$ and $z_{2}=g \cdot t^{4} \in L_{4} \cdot t^{4}$. If $n=\infty$, let $z_{1}=f \cdot t^{2}$ and $z_{2}=g \cdot t^{3}$. By 
Proposition (4.4) it is sufficient to show that in each case the $z_{i}$ satisfy an irreducible relation in $L_{d} \cdot t^{d}$.

$$
\begin{array}{r}
\{1 ; 2\}: z_{2}^{2}-4 z_{1}^{3}+g_{2} z_{0}^{8} z_{1}+g_{3} z_{0}^{12}=0, \\
\{1 ; 3\}: z_{2}^{2}-4 z_{0} z_{1}^{3}+g_{2} z_{0}^{7} z_{1}+g_{3} z_{0}^{10}=0, \\
\{1 ; 4\}: z_{0} z_{2}^{2}-4 z_{1}^{3}+g_{2} z_{0}^{6} z_{1}+g_{3} z_{0}^{9}=0, \\
\{1 ; \infty\}: z_{2}^{2}-4 z_{1}^{3}+g_{2} z_{0}^{4} z_{1}+g_{3} z_{0}^{6}=0,
\end{array}
$$

where $g_{2}, g_{3}$ are complex numbers so that $g_{2}^{3}-27 g_{3}^{2} \neq 0$. These can be seen to be irreducible by considering them as polynomials in $z_{2}$.

$\{1 ; \sigma\}, \sigma=2$ or 3 : These can be explicitly constructed as above using the fact that on an elliptic curve $X$ any divisor of degree $n$ is linearly equivalent to a divisor of the form $n p$ for some $p \in X$ [W1].

All of the groups on Table 1 with $\sigma>0$ are seen to have $L_{G}$ generated by $\leqslant 3$ elements by elementary application of Proposition (4.4), Remark (4.5), (A.1) and (A.2). For example in the case of signature $\{0 ; 2,2,2, \infty\} L$ has two generators $z_{0}$, $z_{1} \in L_{2}$ and one generator $z_{2} \in L_{3}$. Now $z_{0}$ and $z_{1}$ generate the quotient field of $L_{G}$, hence Remark (4.5)(2) is satisfied. As for Remark (4.5)(1), there are clearly no relations in $L_{1}, L_{2}$ or $L_{3}$. Applying (A.2) with $i=r=2$ we see there are no relations in $L_{4}$ and applying (A.1) with $i=3$ and $j=2$ we see there are no relations in $L_{5}$.

Finally, we consider the groups with $g=\sigma=0$. We number the branch points $p_{1}, \ldots, p_{r} \in X=\hat{\mathbf{C}}$, the Riemann sphere. We may assume $p_{1}=0, p_{2}=1$ and $p_{3}=\infty \in \hat{\mathbf{C}}$ and the canonical divisor is $K=-2 \cdot p_{1}$.

$\{0 ; 2,2,2,2,3\}:$ In this case $L_{1}=\{0\}, L_{2}=L\left(-3 \cdot 0+1+\infty+p_{4}+p_{5}\right)$. The latter is generated by

$$
z_{0}=x^{3} /(x-1)\left(x-p_{4}\right)\left(x-p_{5}\right)
$$

and

$$
z_{1}=x^{4} /(x-1)\left(x-p_{4}\right)\left(x-p_{5}\right) .
$$

Rearrange the subscripts so that $e_{1}=3$. Then $L_{3}=L\left(-4 \cdot 0+1+\infty+p_{4}+p_{5}\right)$ is generated by $z_{2}=x^{4} /(x-1)\left(x-p_{4}\right)\left(x-p_{5}\right)$. Clearly Remark (4.5)(2) is satisfied. To verify Remark (4.5)(1) we can easily see using (A.1) and (A.2) that the only possible relation of degree $<d=8$ is in degree 6 . The monomials in $L_{6}$ are $z_{2}^{2}, z_{0}^{3}$, $z_{0}^{2} z_{1}, z_{0} z_{1}^{2}$ and $z_{1}^{3}$. At the point 0 these functions have a zero of order $8,9,10,11$ and 12 respectively. Hence by (4.6.1) they must be linearly independent. Thus Remark (4.5)(1) is satisfied and we have the desired result.

$\{0 ; 2,2,2,2,2\}$ : Let $L_{2}=\left\langle z_{0}, z_{1}\right\rangle$ and $L_{5}=\left\langle z_{2}\right\rangle$. Several applications of (A.1) and (A.2) show that Remark (4.5)(1) and (2) are satisfied.

$\{0 ; 2,3,3,3\}$ : Let $e_{1}=2$. Then $L_{2}=L\left(-3 \cdot 0+1+\infty+p_{4}\right)$ and $L_{3}=$ $L\left(-5 \cdot 0+2 \cdot 1+2 \infty+2 p_{4}\right)$. Let

$$
z_{0}=x^{3} /(x-1)\left(x-p_{4}\right) \in L_{2}, \quad z_{1}=x^{5} /(x-1)^{2}\left(x-p_{4}\right)^{2}
$$


and

$$
z_{2}=x^{6} /(x-1)^{2}\left(x-p_{4}\right)^{2}
$$

Then $L_{2}=\left\langle z_{0}\right\rangle$ and $L_{3}=\left\langle z_{1}, z_{2}\right\rangle$. Clearly Remark (4.5)(2) is satisfied. Now by (A.1) the only possible nontrivial relations of degree $<d=9$ are in $L_{6}$ or $L_{8}$. The monomials in $L_{6}$ are $z_{0}^{3}, z_{1}^{2}, z_{1} z_{2}, z_{2}^{2}$. At the point 0 these functions have a zero of order 9, 10, 11 and 12 respectively. Hence they are linearly independent. A similar calculation in $L_{8}$ shows that Remark (4.5)(1) is satisfied.

$\{0 ; 2,2,3,4\}:$ Let $e_{1}=2, e_{2}=2, e_{3}=3, e_{4}=4$ and define

$$
z_{0}=x^{3} /(x-1)\left(x-p_{4}\right) \in L_{2}, \quad z_{1}=x^{5} /(x-1)\left(x-p_{4}\right)^{2} \in L_{3}
$$

and

$$
z_{2}=x^{7} /(x-1)^{2}\left(x-p_{4}\right)^{3}
$$

Then $L_{2}=\left\langle z_{0}\right\rangle, L_{3}=\left\langle z_{1}\right\rangle, L_{4}=\left\langle z_{0}^{2}, z_{2}\right\rangle$ and $L_{5}=\left\langle z_{0} z_{1}\right\rangle$. Now $L_{6}$ contains the monomials $z_{0}^{3}, z_{1}^{2}$ and $z_{0} z_{2}$. At the point $\infty$ these have poles of order 3,4 and 3 respectively. Thus any relation is of the form $\alpha z_{0}^{3}+\beta z_{0} z_{2}=0$. But any such relation factors, and hence is a consequence of a relation of lower degree. This is impossible. There are no relations of degree 7 or 9 by Proposition (A.1). Finally, the monomials of degree 8 are $z_{0}^{4}, z_{0}^{2} z_{2}, z_{0} z_{1}^{2}$ and $z_{2}^{2}$. At $p_{4}$ these have poles of order $4,4,5$ and 6 respectively. It follows easily that there is no relation of degree 8 .

$\{0 ; 2,2,3,3\}$ : Let $L_{2}=\left\langle z_{0}\right\rangle, L_{3}=\left\langle z_{1}\right\rangle$. Then $z_{0}^{3}$ and $z_{1}^{2}$ are linearly independent since $z_{0}^{3}$ has a pole of order 3 at $\infty$ and $z_{1}^{2}$ has a pole of order 4. Using Table 4 we see there is a $z_{2} \in L_{6}$ so that $L_{6}=\left\langle z_{0}^{3}, z_{1}^{2}, z_{2}\right\rangle$. Then the fact that $L_{G}$ is an integral domain and repeated application of (A.1) shows there is no relation among the $z_{i}$ of degree $<12$. Thus Remark (4.5) and Proposition (4.4) imply that the $z_{i}$ generate $L_{G}$.

$\{0 ; 2,2,2,5\}:$ Let $e_{4}=5$. Then $L_{2}=\left\langle z_{0}=x^{3} /(x-1)\left(x-p_{4}\right)\right\rangle, L_{4}=\left\langle z_{0}^{2}, z_{1}\right.$ $\left.=x^{6} /(x-1)^{2}\left(x-p_{4}\right)^{3}\right\rangle, L_{5}=\left\langle z_{2}=x^{8} /(x-1)^{2}\left(x-p_{4}\right)^{4}\right\rangle$. Applying Propositions (A.1) and (A.2) we see that the only possible relation of degree $<d=12$ is in degree 10. Now the monomials of degree 10 are $z_{0}^{5}, z_{0}^{3} z_{1}, z_{0} z_{1}^{2}$ and $z_{2}^{2}$. At $p_{4}$ these have poles of order $5,6,7$ and 8 respectively. Thus there can be no relation among them. Thus Remark (4.5)(1) is satisfied. Clearly (4.5)(2) holds, hence we get the desired result by Proposition (4.4).

$\{0 ; 2,2,2,4\}$ and $\{0 ; 2,2,2,3\}$ : These two cases follow easily from Propositions (A.1), (A.2) and (4.4).

Consider the groups with $g=\sigma=0$ and $r=3$. In this case there is, up to conjugacy, a unique group for each signature. We can obtain our results by using Remark (4.5) as before. Alternately one can prove that the relation of degree $d$ must be irreducible and apply Proposition (4.4) directly. A third proof can be developed using the theory of Seifert bundles. The crucial fact in that proof is that there is a unique singularity with Seifert invariants $\left\{0 ; b ;\left(\alpha_{1}, \beta_{1}\right),\left(\alpha_{2}, \beta_{2}\right),\left(\alpha_{3}, \beta_{3}\right)\right\}$ [O-W]. 


\section{Appendix.}

Proposition (A.1). Suppose $k$ is a field, $L$ is a graded $k$-algebra, $L$ is an integral domain, $z_{n} \in L_{q_{n}}$ for $n=0,1,2$ and

$$
\mathscr{P}_{L^{(t)}}=\left(1-t^{d}\right) /\left(\prod_{n} 1-t^{q_{n}}\right) \text {. }
$$

Suppose we are given natural numbers $i$ and $j$ and $d$ so that $i+j<d, \operatorname{dim} L_{i}=1$, $\operatorname{dim} L_{j}=L_{i+j}$, and there are no relations of degree $j$ or $i$ (among the $\left.z_{n}\right)$. Then there are no relations of degree $j+i$.

Proof. Let $S=K\left[Z_{0}, Z_{1}, Z_{2}\right]$, define $f: S \rightarrow L$ by $f\left(Z_{n}\right)=z_{n}$ and let $I=$ kernel $f$. If we grade $S$ by letting degree $Z_{n}=q_{n}$, for $n=0,1,2$ then $f$ is a graded homomorphism of degree 0 . Note that $\mathscr{P}_{S}(t)=1 /\left(\Pi_{n} 1-t^{q_{n}}\right)$. Let $X \in S_{i}$ be any nonzero element and $x=f(X)$. Then $x \neq 0$ since there are no relations of degree $i$. Then we have a commutative diagram with exact rows

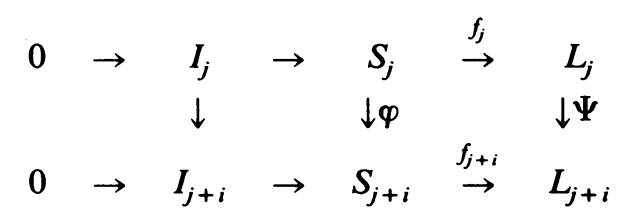

where $\varphi(z)=X Z$ and $\Psi(z)=x z$. Note that no relations of degree $j$ means $I_{j}=\{0\}$. Moreover $\operatorname{dim} S_{j}=$ coefficient of $t_{j}$ in $\mathscr{P}_{S}=$ coefficient of $t^{j}$ in $\mathscr{P}_{L}=$ $\operatorname{dim} L_{j}$. Hence $f_{j}$ is an isomorphism. Now $\Psi$ is one-one since $L$ is a domain; thus $\operatorname{dim} L_{j}=\operatorname{dim} L_{j+i}$ implies $\Psi$ is an isomorphism. Thus $f_{j+i}$ is onto. But as above, $\operatorname{dim} L_{j+i}=\operatorname{dim} S_{j+i}$ and hence $I_{j+i}=\{0\}$.

Proposition (A.2). If $L$ is a graded integral domain over an algebraically closed field $k$ and $x, y \in L_{i}$ are linearly independent, then for all $r \geqslant 1, x^{r}, x^{r-1} y, \ldots, y^{r}$ are linearly independent in $L_{r i}$.

Proof. Suppose $a_{0} x^{r}+a_{1} x^{r-1} y+\cdots+a_{r} y^{r}=0$. Let $t$ be an indeterminate and let $f(t)=\sum_{i=0}^{r} a_{i} t^{i}$. Then $f(t)$ factors into linear factors over $k$,

$$
f(t)=\prod_{i=1}^{r}\left(\alpha_{i} t-\beta_{i}\right) .
$$

But then $\prod_{i=1}^{r}\left(\alpha_{i} y-\beta_{i} x\right)=\sum_{i=0}^{r} a_{i} x^{r-i} y^{i}=0$ and hence $\alpha_{i} y-\beta_{i} x=0$ for some $i$. This implies $\alpha_{i}=\beta_{i}=0$ and hence $a_{i}=0$, for all $i$.

\section{BIBLIOGRAPHY}

[A-M] M. Atiyah and I. MacDonald, Introduction to commutative algebra, Addison-Wesley, Reading, Mass., 1969.

[C-R] P. Connor and F. Raymond, Holomorphic Seifert fiberings, Proc. Second Conf. Compact Transformation Groups, Lecture Notes in Math., vol. 299, Springer-Verlag, Berlin, 1972, pp. 1-75.

[C] J. Carrell, Holomorphically injective complex toral actions, Proc. Second Conf. Compact Transformation Groups, Lecture Notes in Math., vol. 299, Springer-Verlag, Berlin, 1972, pp. 205-236.

[D1] I. Dolgachev, Automorphic forms and quasi-homogeneous singularities, Functional Anal. Appl. 9 (1975), 149-151.

[D2] , Automorphic forms and quasi-homogeneous singularities (preprint). 
[G] R. Gunning, Lectures on modular forms, Notes by Armand Brumer, Princeton Univ. Press, Princeton, N. J., 1962.

[K] I. Kra, Automorphic forms and Kleinian groups, Math. Lecture Notes Series, Benjamin, Reading, Mass., 1972.

[M] J. Milnor, On the 3-dimensional Brieskorn manifolds, $M(p, q, r)$, Knots, Groups and 3-Manifolds (Papers dedicated to the memory of R. H. Fox), Ann. of Math. Studies, no. 84, Princeton Univ. Press, Princeton, N. J., 1975, pp. 175-225.

[O-W] P. Orlik and P. Wagreich, Algebraic surfaces with $k^{*}$-action, Acta Math. 138 (1977), 43-81.

[S-D] B. Saint-Donat, On Petri's analysis of the linear system of quadrics through a canonical curve, Math. Ann. 206 (1973), 157-175.

[S] G. Springer, Introduction to Riemann surfaces, Addison-Wesley, Reading, Mass., 1957.

[W1] P. Wagreich, Elliptic singularities of surfaces, Amer. J. Math. 92 (1970), 419-454.

[W2] __ Automorphic forms and singularities with $\mathbf{C}^{*}$-action, Illinois J. Math. (to appear).

[Z] O. Zariski and P. Samuel, Commutative algebra. I, Van Nostrand, Princeton, N. J., 1959.

Department of Mathematics, University of Illinois at Chicago Circle, Chicago, Illinois 60680 